\title{
DETERIORO COGNITIVO Y TRASTORNO NEURODEGENERATIVO EN PERSONAS CON DISCAPACIDAD INTELECTUAL ${ }^{1}$
}

\section{Cognitive impairment and neurodegenerative disorder in people with intellectual disability}

\author{
Judith Domínguez García \\ Universidad de Salamanca. Instituto Universitario de Integración en la Comunidad. Facultad \\ de Psicología. Avda. de la Merced, 109-131. 37005 Salamanca \\ jdominguez@usal.es \\ Patricia Navas MaChO \\ Universidad de Salamanca. Instituto Universitario de Integración en la Comunidad
}

Recepción: 13 de noviembre de 2017

Aceptación definitiva: 14 de diciembre de 2017

Resumen: El objetivo de este estudio es examinar la posible existencia de deterioro cognitivo en una muestra de personas con discapacidad intelectual (DI) mayores de 35 años, con el fin de realizar un diagnóstico temprano. Participaron 36 personas con DI de edades comprendidas entre los 35 y los 61 años del centro Fundación Personas (Asprosub) de Zamora. Se aplicó la adaptación española (Esteba-Castillo et al., 2013) del Cambridge Examination for mental disorders of older people with Down's syndrome and others with intellectual disabilities, CAMDEX-DS (Ball, Dodd, Holland, Huppert y Treppner, 2006). Se empleó la batería neuropsicológica Cambridge Cognitive Examination (CAMCOG), prueba integrada en el CAMDEX-DS orientada a valorar los aspectos cognitivos que pudieran estar alterados, $\mathrm{y}$, en el caso de sospecha de deterioro cognitivo, se realizó una entrevista con un profesional con el fin de obtener información sobre posibles cambios que hubieran podido surgir en el comportamiento de la persona

1 Este artículo se ha escrito en el marco del proyecto de investigación I+D PSI2015-64517-R. financiado por el Ministerio de Economía y Competitividad dentro de las ayudas para la realización de proyectos de investigación dentro del Programa Estatal de Investigación, Desarrollo e Innovación Orientada a los Retos de la Sociedad, Convocatoria 2015. 


\section{DETERIORO COGNITIVO Y TRASTORNO NEURODEGENERATIVO EN PERSONAS CON DISCAPACIDAD INTELECTUAL \\ JUDITH DOMÍNGUEZ GARCÍA Y PATRICIA NAVAS MACHO}

con DI. Los resultados indicaron que el $22 \%$ de la muestra obtuvo puntuaciones que indicaron riesgo de deterioro cognitivo. Según los informantes, estas personas presentaban cambios conductuales y de personalidad, así como cambios en las habilidades de la vida diaria. No se encontraron diferencias significativas en lo que a presencia de deterioro cognitivo se refiere en función de la edad. En cambio, al analizar la etiología y el grado de DI (leve o moderado) los resultados indicaron que las personas con síndrome de Down obtuvieron puntuaciones significativamente más bajas (indicativas de deterioro cognitivo) que aquellas cuyas DI no se debe a esta etiología. Estos mismos resultados se observaron en las personas con DI moderada frente a aquellas cuyas limitaciones son más leves. Se discuten los resultados obtenidos y se señalan las limitaciones del estudio.

Palabras clave: discapacidad intelectual; prevalencia; síndrome de Down; deterioro cognitivo.

АвтRAст: The aim of this study is to explore the prevalence of cognitive impairment in a sample of individuals with intellectual disability (ID) aged 35 years old and over, with the purpose of establishing an early diagnosis. Participants were 36 individuals with ID whose ages ranged from 35 to 61 years old, all of them receiving services at Fundación Personas (Asprosub) in Zamora. The Spanish version (Esteba-Castillo et al., 2013) of the Cambridge examination for mental disorders of older people with Down syndrome and others with intellectual disabilities, CAMDEX-DS (Ball, Dodd, Holland, Huppert and Treppner, 2006) was applied. As part of this assessment instrument, the neuropsychological Cambridge Cognitive Examination battery, CAMCOG, aimed at measuring cognitive impairment, was applied; when scores indicated a suspicion of cognitive impairment, an interview with a professional was carried out to obtain information about possible changes that could have arisen in the behavior of our participants. Results indicated that $22 \%$ of the sample has a risk of cognitive impairment. According to the informants, these individuals have manifested behavioral and personality changes, and changes in their abilities of daily life. No significant differences were found in the diagnosis of cognitive impairment as a function of age. On the other hand, when analyzing the etiology and the degree of ID (mild or moderate), people with Down syndrome scored significantly lower than those whose ID was not due to this etiology. On the other hand, individuals with moderate ID presented a higher ratio of cognitive impairment than those with mild ID. The results obtained are discussed and the limitations of the study are indicated.

KEY WORDS: intellectual disability; prevalence; Down syndrome; cognitive impairment.

\section{Introducción}

EgÚn los Datos De la EnCUESTA SOBRe Discapacidad, Autonomía Personal y
Situaciones De DePEndencia, EDAD (Instituto Nacional de Estadística, 2008),
la esperanza de vida de la población española ronda los 80 años. Quizá debido a
su carácter inevitable, la vejez junto con el deterioro asociado que puede suponer, no 
ha dejado de preocupar a los investigadores (Barrio del Campo, Árias, Ruiz Fernández y Vicente Castro, 2007).

Los avances en medicina, la mejor prestación de servicios a personas que envejecen y los cambios en el estilo de vida, entre otros factores, han dado lugar a un incremento en la esperanza de vida, incremento que en los últimos años se ha trasladado también al colectivo de personas con discapacidad intelectual (DI), llegando a tener una expectativa de vida muy similar a la de la población en general (Esteba-Castillo, Ribas, Baró y Novell, 2006; Silverman, Zigman, Krinsky-McHale, Ryan y Schupf, 2013; Borras, 2015). En el caso específico de las personas con síndrome de Down (SD), diversos estudios han demostrado un incremento en la esperanza de vida de este colectivo desde la década de los 60 (en un 456\%), probablemente relacionado con los avances en la atención médica y la desinstitucionalización (Presson et al., 2013).

Cuando hablamos de envejecimiento, no podemos evitar pensar en las patologías asociadas al mismo, las cuales han visto incrementada su prevalencia conforme ha ido aumentando la esperanza de vida. En el caso específico de las personas con DI, se observa además una mayor vulnerabilidad frente al desarrollo de patologías específicas relacionadas con el envejecimiento, siendo una de ellas el deterioro cognitivo (Heller, 2009; Silverman et al., 2013; Tassé et al., 2016).

El trastorno neurocognitivo mayor según la Asociación Americana de Psiquiatría, APA (2013), es un síndrome adquirido crónico de origen orgánico en el que las funciones mentales superiores (memoria, pensamiento, orientación, comprensión, cálculo, aprendizaje, lenguaje y juicio) están dañadas, interfiriendo en la independencia de la persona.

En el caso específico de las personas con SD, la trisomía del gen 21 incluye material genético que es precipitante de cambios neurobiológicos que se observan en el trastorno neurocognitivo conocido como demencia tipo Alzheimer, incrementando la vulnerabilidad de este colectivo a experimentar esta patología (Díaz, Gómez y Alcedo, 2016). No obstante, la mayoría de las personas con DI reciben un diagnóstico de deterioro cognitivo cuando este ya está en etapas avanzadas, lo que dificulta que se puedan poner en marcha medidas para tratar de ralentizar el proceso de la enfermedad. El diagnóstico tardío responde, entre otras variables, a la dificultad para identificar los posibles primeros síntomas del deterioro cognitivo en un colectivo que presenta de por sí un funcionamiento intelectual significativamente limitado (Janicki y Dalton, 2000; Bell, Turnbull y Kidd, 2009; McCarron, McCallion, Reilly y Mulryan, 2014), por lo que los posibles primeros síntomas del deterioro pueden llegar a confundirse con la etiología propia de la discapacidad. Además, es necesario remarcar que la mayor parte de las baterías neuropsicológicas para la valoración del estado mental, la cognición y las capacidades funcionales han sido estandarizadas en población en general (Makary et al., 2015; Walsh et al., 2015), careciendo de baremos específicos para población con DI y pudiendo por tanto dar lugar a la emisión de falsos negativos y falsos positivos en el proceso de valoración diagnóstica en este colectivo.

Con objeto de facilitar la identificación de deterioro cognitivo en esta población, en la actualidad se plantea que, a la hora de realizar la evaluación, es necesario explorar 
múltiples áreas de funcionamiento. Livingston, King, Hassiotis y Stydom (2007) afirman que, para poder realizar una correcta evaluación ante un posible caso de deterioro cognitivo, es necesario realizar una exploración neuropsicológica, un examen físico y un examen del estado mental de la persona con DI. Este último se realiza con el fin de descartar que los síntomas sean debidos a otra patología psiquiátrica (Bell et al., 2009). Una buena evaluación, además, requiere el estudio longitudinal del paciente que permita comparar el funcionamiento base de la persona con el presente, así como su desarrollo conductual en un período de mínimo 6 meses (Díaz et al., 2016). Este estudio longitudinal es necesario ya que en población general es relativamente fácil conocer si se ha producido un deterioro en el funcionamiento habitual de la persona, pero en el caso de adultos mayores con DI o SD esta tarea es mucho más difícil ya que el registro de información sobre las personas con discapacidad depende de múltiples factores, como son la participación de las familias, la ausencia de registros previos acerca del funcionamiento de la persona o la escasa formación de los profesionales sanitarios a la hora de identificar posibles deterioros en la persona con DI, entre otros.

A las dificultades anteriormente nombradas, se une la variabilidad en la manifestación de los síntomas del deterioro cognitivo en este colectivo. En población sin discapacidad, el trastorno neurodegenerativo suele comenzar con problemas relacionados con la memoria para continuar con los problemas conductuales. Sin embargo, en personas con DI, los problemas de conducta suelen preceder a los problemas de memoria (Janicki, Heller, Seltzer y Hogg, 1996; Sheehan et al., 2015). Es por este motivo que, en ocasiones, el diagnóstico no se realiza hasta que el deterioro ya está avanzado, ya que los problemas conductuales pueden llegar a confundirse con características propias de la DI.

Por todas las razones comentadas, es necesario remarcar la importancia de establecer una serie de pasos a la hora de evaluar a personas con DI con sospecha de deterioro cognitivo. Este proceso debe incluir, según Moran et al. (2013), los siguientes aspectos: (a) conocer la historia médica y psiquiátrica; (b) realizar una descripción del funcionamiento base de la persona y realizar un seguimiento longitudinal del mismo; (c) realizar un inventario de problemas comórbidos al deterioro cognitivo; (d) revisar todos los medicamentos que toma la persona con el fin de descartar efectos secundarios de los mismos; (e) obtener el historial familiar para conocer posibles antecedentes de deterioro cognitivo; (f) evaluar posibles cambios en el entorno del individuo que hayan podido precipitar un deterioro en su funcionamiento; (g) revisar el ambiente de la persona con el fin de conocer su red de apoyo, incluyendo el nivel de apoyos; y (h) realizar una puesta en común de toda esta información (Moran et al., 2013).

El curso exacto y la presentación del deterioro cognitivo en DI y SD sigue siendo desconocido. Esto es debido a que, aunque existe una amplia disponibilidad de pruebas para el diagnóstico de deterioro cognitivo en población sin discapacidad, estas pruebas no presentan baremos adaptados para personas con DI y las pruebas específicas que existen presentan dificultades para detectar esta patología en su curso inicial. Por esta razón, se recomienda investigar los cambios de comportamiento o cognitivos 
en adultos con SD de más de 30 años, así como estar atentos a posibles cambios de conducta en adultos con DI sin etiología específica, tanto para mejorar el proceso de diagnóstico como su calidad de vida. Otras dificultades que aparecen debidas a la falta de instrumentación específica para la población DI son las limitaciones en las propiedades psicométricas, es decir, que estos instrumentos se caracterizan por presentar una baja fiabilidad y validez en este tipo de población. Todo lo dicho anteriormente pone de manifiesto la necesidad de desarrollar nuevas herramientas de evaluación que permitan superar las limitaciones encontradas.

A la hora de evaluar el funcionamiento cognitivo de la persona con DI encontramos varios test como el Dementia Questionnarie for People with Learning Disabilities de Eurlings, Evenhuis y Kengen (2006). También hay que destacar el Dementia Screening Questionnaire for individuals with ID de Deb, Hare, Prior y Bhaumik (2007) y el Adaptive Behavior Dementia Quiestionnaire de Prasher, Farooq y Holder (2004) (citados por Bell et al., 2009; Díaz et al., 2016; McCarron et al., 2014; y Sheehan et al., 2015), pero entre todos ellos destacamos The Cambridge Examination for Mental Disorders of Older People with Down's Syndrome and Others with Intellectual Disabilities (CAMDEX-DS) de Ball, Dodd, Holland, Huppert y Treppner (2006), ya que, además de ser el único instrumento en lengua española específicamente dirigido a población con DI, proporciona estructura y apoyo para la buena práctica en el diagnóstico e investigación de esta patología ya que evalúa memoria, función intelectual general, juicio, rendimiento general, función cortical superior y personalidad, además de haber demostrado, en su versión original, buena fiabilidad y validez predictiva (Díaz, Gómez y Alcedo, 2016). Este instrumento surge a partir de la prueba dirigida a población general Cambridge Examination for Mental Disorders of Older People (CAMDEX) revisada en 1998 por Huppert, López, Mounthoy y Roth (1998).

El CAMDEX-DS ha sido adaptado a población española por Esteba-Castillo et al. (2013) y ha sido diseñado para ser administrado en entornos comunitarios por profesionales de la salud mental o en el contexto de una investigación. Consta de 3 partes: (1) el inventario CAMDEX-DS, con el que se pretende delimitar, a partir de la entrevista con un profesional, el estado cognitivo, funcional y neuropsiquiátrico del paciente; (2) la evaluación del paciente a partir de una entrevista clínica; y (3) la batería de exploración neuropsicológica Cambridge Cognitive Examination (CAMCOG), que es aplicada por un profesional a partir de la información proporcionada por la persona con DI. Esta breve batería fue diseñada para evaluar los déficits cognitivos especificados en los criterios diagnósticos operativos para demencia y evalúa orientación, lenguaje (comprensión y expresión), memoria (nuevos aprendizajes, memoria a corto y largo plazo), atención, praxis (dibujo de figuras complejas y la capacidad para llevar a cabo tareas complejas), pensamiento abstracto y percepción. No obstante, y a pesar de reconocer el esfuerzo de los adaptadores por facilitar el acceso en lengua española de este instrumento, sería necesario contar con estudios de mayor tamaño muestral que den cuenta de sus propiedades psicométricas. Este aspecto será tratado con mayor detalle en la discusión con ánimo de identificar necesarias líneas de investigación futuras. 
Además de los instrumentos anteriormente mencionados, a la hora de realizar un diagnóstico de posible deterioro cognitivo en personas con DI, es importante tener en cuenta la guía clínica para el diagnóstico de enfermedades mentales en personas con discapacidad intelectual (Diagnostic Manual-Intellectual Disability, DM-ID), actualmente en su segunda edición (DM-ID 2), desarrollada a partir de la adaptación de los criterios diagnósticos del DSM-5 para esta población (Fletcher, Barnhill y Cooper, 2017). Como criterios del trastorno neurodegenerativo se incluyen: (1) presentar un declive cognitivo con respecto a un nivel previo de mayor desempeño; (2) los déficits cognitivos son suficientes para interferir con la independencia; (3) no ocurren exclusivamente en el contexto de un delirium; y (4) no son atribuibles de forma primaria a la presencia de otros trastornos mentales.

Teniendo en cuenta la relevancia del diagnóstico precoz, el propósito de este estudio se centra en explorar la posible existencia de trastorno neurocognitivo mayor en una muestra de personas con DI mayores de 35 años $(\mathrm{N}=36)$, con el fin de realizar un diagnóstico temprano a partir de la versión española del CAMDEX-DS (EstebanCastillo et al., 2013).

Este objetivo general se puede operacionalizar en los siguientes objetivos específicos:

(1) Conocer si la edad constituye una variable que pudiera actuar como buen predictor del diagnóstico de deterioro cognitivo en personas con DI.

(2) Conocer si existen diferencias estadísticamente significativas entre población con DI de etiología no especificada y población con síndrome de Down en el diagnóstico de deterioro cognitivo a partir de las puntuaciones obtenidas en la batería neuropsicológica CAMCOG.

(3) Conocer si existen diferencias estadísticamente significativas, a partir de las puntuaciones obtenidas en la batería neuropsicológica CAMCOG, en función del grado de DI (leve o moderada) en lo que a diagnóstico de deterioro cognitivo se refiere.

(4) Comprobar si las personas que presentan puntuaciones indicativas de sospecha de deterioro cognitivo a través del CAMCOG presentaron problemas de conducta como primera manifestación de esta etiología.

(5) Comprobar si las personas con sospecha de deterioro cognitivo a partir del CAMCOG cumplen los criterios de trastorno neurocognitivo mayor recogidos en el DM-ID-2 (Fletcher et al., 2017).

A partir de estos objetivos se pretende verificar las siguientes hipótesis:

(1) La edad constituye un buen predictor de deterioro cognitivo en personas con DI y SD.

(2) Las personas con síndrome de Down obtendrán puntuaciones significativamente más bajas, indicativas de sospecha de deterioro cognitivo, que aquellas personas con DI que no presenten esta etiología.

(3) Aquellas personas con DI moderada obtendrán puntuaciones significativamente más bajas, indicativas de sospecha de deterioro cognitivo, que aquellas con DI leve. 


\section{Método}

\subsection{Participantes}

La población objeto de este estudio son personas con discapacidad que reúnen los siguientes requisitos: personas con un diagnóstico confirmado de DI con edad superior a los 35 años y que presenten un nivel intelectual que permita responder a las preguntas que se le han de plantear a través del CAMDEX-DS (Esteban-Castillo et al., 2013). Además, no deben presentar otro tipo de discapacidad que pueda dificultar responder a las preguntas.

Para seleccionar a los participantes se realizó un muestro intencional o de conveniencia, en función de la accesibilidad y proximidad de los posibles participantes del estudio, todos ellos usuarios de la Fundación Personas de Zamora (antes conocida como Asprosub) y con un diagnóstico previo confirmado de DI. Esta organización presta servicios a un total de 194 personas con DI, de las cuales 40 cumplieron los criterios de inclusión, obteniendo el consentimiento informado para participar en la investigación de un total de 36 .

La muestra se distribuyó de manera equitativa en lo que a sexo se refiere. Sus edades oscilaron entre los 35 y los 61 años $(M=47,81, D T=7,96)$. En la Tabla 1 se recogen todas las características sociodemográficas de la muestra.

\begin{tabular}{|c|c|c|c|c|c|c|}
\hline & \multicolumn{4}{|c|}{ Nivel de discapacidad intelectual (DI) } & \multirow{2}{*}{\multicolumn{2}{|c|}{ TOTAL }} \\
\hline & & EVE & DI & ERADA & & \\
\hline & $\mathrm{N}$ & $\%$ & $\mathrm{~N}$ & $\%$ & $\mathrm{~N}$ & $\%$ \\
\hline $\begin{array}{l}\text { Género } \\
\text { Varón } \\
\text { Mujer }\end{array}$ & $\begin{array}{l}11 \\
12 \\
\end{array}$ & $\begin{array}{r}47,80 \\
52,20 \\
\end{array}$ & $\begin{array}{l}7 \\
6 \\
\end{array}$ & $\begin{array}{r}53,80 \\
46,20 \\
\end{array}$ & $\begin{array}{l}18 \\
18 \\
\end{array}$ & $\begin{array}{l}50 \\
50 \\
\end{array}$ \\
\hline $\begin{array}{c}E d a d \\
35-40 \\
41-44 \\
45-48 \\
49-53 \\
54-57 \\
58+\end{array}$ & $\begin{array}{l}3 \\
2 \\
3 \\
6 \\
4 \\
5\end{array}$ & $\begin{array}{c}13 \\
8,70 \\
13 \\
26,10 \\
17,40 \\
21,70\end{array}$ & $\begin{array}{l}5 \\
3 \\
1 \\
2 \\
0 \\
2 \\
\end{array}$ & $\begin{array}{c}38,50 \\
23,10 \\
7,70 \\
15,40 \\
0 \\
15,40\end{array}$ & $\begin{array}{l}8 \\
5 \\
4 \\
8 \\
4 \\
7\end{array}$ & $\begin{array}{l}22,20 \\
13,90 \\
11,10 \\
22,20 \\
11,10 \\
19,40\end{array}$ \\
\hline $\begin{array}{c}\text { Etiología } \\
\text { Síndrome de Down } \\
\text { DI de etiología no especificada }\end{array}$ & $\begin{array}{r}7 \\
16 \\
\end{array}$ & $\begin{array}{l}30,40 \\
69,60 \\
\end{array}$ & $\begin{array}{r}11 \\
2 \\
\end{array}$ & $\begin{array}{l}84,60 \\
15,40 \\
\end{array}$ & $\begin{array}{l}18 \\
28 \\
\end{array}$ & $\begin{array}{l}50 \\
50 \\
\end{array}$ \\
\hline $\begin{array}{c}\text { Entorno residencial } \\
\text { Familia } \\
\text { Residencia } \\
\text { Vivienda tutelada } \\
\end{array}$ & $\begin{array}{r}12 \\
5 \\
6 \\
\end{array}$ & $\begin{array}{l}52,20 \\
21,70 \\
26,10\end{array}$ & $\begin{array}{r}10 \\
1 \\
2\end{array}$ & $\begin{array}{r}76,90 \\
7,70 \\
15,40 \\
\end{array}$ & $\begin{array}{r}22 \\
6 \\
8\end{array}$ & $\begin{array}{l}16,70 \\
61,10 \\
22,20\end{array}$ \\
\hline $\begin{array}{c}\text { Ocupación } \\
\text { Centro especial de empleo (CEE) } \\
\text { Servicio de terapia ocupacional (STO) }\end{array}$ & $\begin{array}{r}2 \\
21\end{array}$ & $\begin{array}{r}8,70 \\
91,30\end{array}$ & $\begin{array}{r}0 \\
13\end{array}$ & $\begin{array}{r}0 \\
100\end{array}$ & $\begin{array}{r}2 \\
34\end{array}$ & $\begin{array}{r}5,60 \\
94,40\end{array}$ \\
\hline
\end{tabular}

(C) Ediciones Universidad de Salamanca / CC BY-NC-ND

Siglo Cero, vol. 49 (1), n. ${ }^{\circ} 265,2018$, enero-marzo, pp. 53-67 


\section{DETERIORO COGNITIVO Y TRASTORNO NEURODEGENERATIVO EN PERSONAS CON DISCAPACIDAD INTELECTUAL \\ JUDITH DOMÍNGUEZ GARCÍA Y PATRICIA NAVAS MACHO}

\subsection{Instrumento}

El instrumento utilizado en el presente trabajo ha sido la versión adaptada al castellano por Esteba-Castillo et al. (2013) del CAMDEX-DS (Ball et al., 2006). Esta escala está estructurada en tres bloques, comentados con anterioridad y que detallaremos a continuación. El primero es el inventario CAMDEX-DS, con el que se pretende delimitar el estado cognitivo, funcional y neuropsiquiátrico del paciente. Consta de:

(1) Una entrevista estructurada con un informante. Se trata de una entrevista clínica que pretende facilitar la recogida de información sobre los síntomas que presenta la persona, así como su historia clínica. Consta de 4 partes: (1) funcionamiento del paciente; (2) deterioro cognitivo y funcional (habilidades de la vida diaria, memoria, orientación, otras habilidades cognitivas, personalidad y autocuidado); (3) estado de salud mental (depresión, ansiedad, trastorno paranoide y delirium); y (4) estado de salud física (discapacidad física, hipotiroidismo, problemas cerebrovasculares y otras enfermedades orgánicas).

(2) Breve entrevista clínica con el paciente que incluye historia clínica, estado de salud mental actual y otra información adicional relacionada con síntomas de demencia.

(3) Batería de exploración neuropsicológica (CAMCOG): breve batería que fue diseñada para evaluar los déficits cognitivos especificados en los criterios diagnósticos operativos de demencia. Evalúa orientación, lenguaje (comprensión y expresión), memoria (nuevos aprendizajes, remota y reciente), atención, praxis (dibujo de figuras complejas y la capacidad para llevar a cabo tareas complejas), pensamiento abstracto y percepción.

El segundo bloque corresponde a una guía para el diagnóstico clínico, que proporciona una serie de ayudas para establecer un diagnóstico adecuado descartando otras enfermedades o trastornos.

El último bloque incluye una serie de directrices para la intervención (Ball, Holland, Huppert, Treppner y Dodd, 2013).

Los estudios realizados hasta la fecha sobre la versión española indican una fiabilidad test-retest e interjueces calculada con el coeficiente kappa de 0,92 y 0,91, respectivamente, y la fiabilidad interna calculada mediante el alfa de Cronbach de 0,93 (Esteban-Castillo et al., 2013).

Puntuaciones más bajas en la batería neuropsicológica se traducen en un mayor riesgo de deterioro cognitivo. El instrumento proporciona puntos de corte en función del grado de discapacidad (p. e., leve o moderada), que indican mayor riesgo de deterioro cognitivo. En DI leve, puntuaciones por debajo de 68 puntos indican sospecha de deterioro, mientras que en el caso de DI moderada el punto de corte se establece en 52 puntos.

El instrumento se puede aplicar de forma completa o aplicar pruebas por separado, aunque lo más correcto sería realizar todas las pruebas para obtener una descripción más detallada de la persona.

La información dada por la batería neuropsicológica, junto a la entrevista estructurada con el informante y empleando la guía para el diagnóstico clínico, nos permiten 
obtener información para poder llevar a cabo un diagnóstico preciso, en función de los criterios de trastorno neurocognitivo mayor recogidos en el DM-ID-2 (Fletcher et al., 2017).

\subsection{Procedimiento}

El procedimiento de recogida de datos se llevó a cabo acudiendo al centro Fundación Personas de Zamora. Se contactó con la psicóloga del centro y se le informó de los objetivos de la investigación. Se presentó en un primer contacto un breve resumen de la investigación, aclarando el propósito del estudio, el instrumento que se iba a utilizar y cómo iba a asegurarse la confidencialidad de los datos. Una vez que el centro autorizó la investigación, se le facilitó una carta con información del estudio para que se la dirigieran a las familias, de modo que estas pudieran dar su consentimiento informado. Esta información fue entregada a las familias por la psicóloga del centro.

La identidad de las personas no figuró en el cuaderno de recogida de datos y se utilizaron códigos personales formados por las iniciales y un número para la identificación de los participantes.

El proceso de administración del instrumento se inició el 1 de marzo de 2017 y terminó el 21 de abril del mismo año. Para esta investigación solo se empleó la batería neuropsicológica CAMCOG y la entrevista a personas con DI, y en aquellos casos en los que se obtuvieron puntuaciones por debajo de los puntos de corte indicados, se llevó a cabo la entrevista con el profesional para así poder obtener más información sobre la persona.

\subsection{Análisis de datos}

Se utilizó el programa estadístico SPSS v. 23 y, dado el reducido tamaño de la muestra, los contrastes se realizaron con un nivel de significación de $\alpha=.05$.

El primer paso fue obtener las puntuaciones totales del CAMCOG. Para ello se sumaron las puntuaciones obtenidas por los participantes en las siete secciones que lo integran (p. e., orientación, lenguaje, memoria, atención, praxis, pensamiento abstracto y percepción).

Para contrastar nuestra primera hipótesis (p .e., conocer si la edad puede considerarse un buen predictor de deterioro cognitivo en personas con DI y SD), se realizó una regresión simple.

Además, y dado el pequeño tamaño de la muestra, se utilizó la prueba U de Mann Whitney para conocer si, en función del grupo de edad al que pertenecen, los participantes obtienen puntuaciones más bajas. Para ello, y debido al pequeño tamaño de la muestra, se dividió a los participantes en dos intervalos iguales (hasta 45 años y por encima de los 45).

Para comprobar si las personas con síndrome de Down puntúan más bajo en el CAMCOG que aquellas con DI de etiología no especificada y para saber si aquellas 


\section{DETERIORO COGNITIVO Y TRASTORNO NEURODEGENERATIVO EN PERSONAS CON DISCAPACIDAD INTELECTUAL \\ JUDITH DOMÍNGUEZ GARCÍA Y PATRICIA NAVAS MACHO}

personas con DI moderada presentarán una ratio mayor de deterioro cognitivo que aquellas con DI leve, volvió a realizarse el contraste no paramétrico U de MannWhitney debido al tamaño de la muestra.

\section{Resultados}

En primer lugar, los resultados cuantitativos que se obtuvieron en el CAMCOG aparecen reflejados en la Tabla 2, en la que se indica el número de participantes que obtuvieron puntuaciones por encima o por debajo del punto de corte en función de su nivel de DI.

TABLA 2. Registro de puntuaciones por encima y debajo de los puntos de corte

\begin{tabular}{|l|c|c|c|}
\hline & DI Leve & DI Moderada & Total \\
\hline Puntuaciones por encima del punto de corte & 14 & 14 & 28 \\
\hline Puntuaciones por debajo del punto de corte & 4 & 4 & 8 \\
\hline Total & 18 & 18 & 36 \\
\hline
\end{tabular}

Una vez obtenidas las puntuaciones, se llevó a cabo una regresión lineal simple, con objeto de identificar si la variable edad puede contribuir a la predicción de las puntuaciones en esta prueba. Se encontró una ecuación de regresión no significativa $[\mathrm{F}(1,34)=, 01, p=.93]$, con un $\mathrm{R}^{2}$ de, 02 .

Se analizó, además, si las personas con edades superiores a los 45 años obtenían puntuaciones significativamente más bajas en el CAMCOG que aquellos que eran más jóvenes (p. e., menores de 45 años), pero podemos ver que las puntuaciones en el CAMCOG de estos últimos $(M d n=70)$ no difieren significativamente de las obtenidas por los mayores de $45(M d n=77)$ [ $U=134,5, z=-, 494, p=, 63]$.

A continuación, se comprobó que las personas con síndrome de Down obtenían puntuaciones significativamente más bajas en la batería neuropsicológica CAMCOG $(M d n=70)$ que aquellas personas que no presentaban esta etiología $(M d n=84,5)[U$ $=94,5 z=-2,14, p<, 05]$.

Además, las personas con DI moderada presentaban puntuaciones significativamente más bajas en el CAMCOG $(M d n=66)$ que aquellos participantes que presentaban DI leve $(M d n=85)$, siendo estas diferencias estadísticamente significativas $[U$ $=44 z=-2,34, p<, 05]$.

Cuando las personas obtuvieron puntuaciones por debajo de los puntos de corte $(\mathrm{N}=8)$, se realizó la entrevista con el informante (profesional de Asprosub) con el fin de obtener más información sobre las mismas. Los cuidadores reportaron que las personas habían presentado dificultades en las actividades de la vida diaria y problemas de conducta surgidos recientemente. En la Tabla 3 se exponen todas las partes de la entrevista y qué porcentaje de personas presentaba dificultades o alteraciones en dichas áreas. Se ha de tener en cuenta que una misma persona puede manifestar varios tipos de dificultades y alteraciones. Como podemos observar y de acuerdo con lo que 
expone la literatura científica, el 100\% de los casos con deterioro cognitivo manifestó cambios conductuales y de personalidad con respecto a su comportamiento habitual.

\begin{tabular}{|l|c|c|}
\hline \multicolumn{2}{|l|}{ TABLA 3. Descripción de los cambios observados a partir de la entrevista con el profesional } \\
\hline & Casos & Porcentaje \\
\hline Habilidades de la vida diaria & 7 & $87,5 \%$ \\
\hline Memoria & 4 & $50 \%$ \\
\hline Orientación & 2 & $25 \%$ \\
\hline Funcionamiento mental general & 3 & $37,5 \%$ \\
\hline Lenguaje & 1 & $12,5 \%$ \\
\hline Percepción & 2 & $25 \%$ \\
\hline Funciones ejecutivas & 3 & $37,5 \%$ \\
\hline Personalidad y conducta & 8 & $100 \%$ \\
\hline Autocuidado & 4 & $50 \%$ \\
\hline Depresión & 1 & $12,5 \%$ \\
\hline Ansiedad & 0 & $0 \%$ \\
\hline Síntomas paranoides & 0 & $0 \%$ \\
\hline Obnubilación/delirium & 4 & $50 \%$ \\
\hline
\end{tabular}

\section{Discusión y conclusiones}

Como objetivo principal, esta investigación se planteaba explorar la posible existencia de deterioro cognitivo o trastorno neurocognitivo mayor en una muestra de personas con DI mayores de 35 años, con el fin de realizar un diagnóstico temprano. En este caso vemos que del total de la muestra $(\mathrm{N}=36), 8$ personas $(22,2 \%)$ obtenían puntuaciones por debajo del punto de corte establecido para confirmar sospecha de deterioro cognitivo y cumplían los criterios diagnósticos establecidos por el manual diagnóstico DM-ID 2, por lo que podríamos concluir que estas 8 personas presentarían un trastorno neurocognitivo mayor.

En relación al primer objetivo específico de este estudio, orientado a conocer si la edad puede ser considerada como un buen predictor de deterioro cognitivo, no hemos podido demostrar que una mayor edad se asocie de manera estadísticamente significativa con sospecha de deterioro cognitivo. Tampoco hemos encontrado diferencias estadísticamente significativas en las puntuaciones obtenidas en la batería CAMCOG entre grupos de edad (menores y mayores de 45 años). Otros estudios (Oliver et al., 2008; Presson et al., 2013) sí manifiestan que existen diferencias en función de la edad en el diagnóstico de deterioro cognitivo. No obstante, hay que remarcar que estas investigaciones tenían un tamaño muestral mucho mayor que el de esta investigación y existía, además, una mayor variabilidad en la muestra. Además, el tamaño limitado de la muestra nos ha impedido realizar otro tipo de análisis (p. e., regresión lineal múltiple) que contemplen la posible contribución de distintas variables (p. e., edad, 
severidad de la DI, entre otras) y su influencia sobre la variable dependiente (p. e., puntuaciones en el CAMCOG).

Otro objetivo del presente trabajo fue conocer si existen diferencias estadísticamente significativas en las puntuaciones obtenidas en el CAMCOG entre población con DI de etiología no especificada y población con síndrome de Down. Los resultados obtenidos ponen de manifiesto que las personas con SD obtenían puntuaciones significativamente más bajas en el CAMCOG que aquellas que no presentaban esta etiología. Las diferencias encontradas entre estas dos poblaciones concuerdan con otros hallazgos como los realizados por Livingston et al. (2007) y pudieran estar relacionadas con la vulnerabilidad genética ya comentada presente en el SD. Diversas investigaciones recogidas en Díaz et al. (2016) mencionan la existencia de una mayor presencia del alelo b4 de la Apolipoproteína E en las personas con SD y deterioro cognitivo (Schupf et al., 1998, citado por Díaz et al., 2016). No obstante, aún queda camino por recorrer en el estudio de los factores de riesgo genéticos asociados a la temprana aparición de deterioro cognitivo (Jones et al., 2013, citado por Díaz et al., 2016).

Los resultados obtenidos ponen también de manifiesto que existen diferencias estadísticamente significativas entre las personas con DI leve y moderada en lo que a presencia de deterioro cognitivo se refiere. Así, las personas con DI moderada presentaron puntuaciones significativamente más bajas. En este sentido cabe destacar que no existen investigaciones en las que se ponga de manifiesto si existe una mayor prevalencia de deterioro cognitivo en personas con DI moderada. Una futura línea de investigación podría orientarse a comparar estas dos poblaciones y ver si existen diferencias en cuanto a diagnóstico y en el propio desarrollo del deterioro cognitivo.

Con respecto a nuestro penúltimo objetivo, comprobar si aquellos con sospecha de deterioro cognitivo a través del CAMCOG presentaban problemas de conducta, pudimos observar que en la totalidad de los casos los informantes manifestaban que las personas habían sufrido cambios en la conducta y en la personalidad. En este caso, los hallazgos concuerdan con lo expuesto en otras investigaciones (Bell et al., 2009; Esteba-Castillo et al., 2006; Sheehan et al., 2015). En muchas ocasiones, la dificultad del diagnóstico radica en que estos cambios se atribuyen a la propia discapacidad de la persona (p. e., efecto eclipsador de la discapacidad). Por ello es necesario remarcar que para poder realizar un buen diagnóstico es necesario contar con una línea base inicial de comportamiento que nos permita determinar cuál es el comportamiento "normal" de cada persona y así poder realizar comparaciones entre dicha línea de base y los registros conductuales que debieran llevarse a cabo en el momento de la evaluación. Instrumentos como la entrevista con el informante y la entrevista con la propia persona con los que cuenta el CAMDEX-DS nos sirven para obtener un perfil sobre las características de cada individuo y compararlos con la línea base con la que ya contábamos. Estos instrumentos, no obstante, siempre han de ir acompañados de un diagnóstico clínico que verifique el cumplimiento de los criterios diagnósticos de deterioro cognitivo adaptados a personas con DI (DM-ID-2).

El último objetivo consistía en verificar que las personas que presentaban puntuaciones de riesgo de deterioro cognitivo, es decir, por debajo de los puntos de corte, cumplían con los criterios diagnósticos de trastorno neurocognitivo mayor recogidos 
en el manual diagnóstico DM-ID 2 (Fletcher et al., 2017). Todas las personas con puntuaciones por debajo de los puntos de corte cumplieron con los 4 criterios presentados en el manual, enunciados con anterioridad, lo que nos permitiría emitir un diagnóstico preciso sobre la presencia del trastorno neurocognitivo mayor.

En cuanto a las limitaciones que presenta esta investigación, hemos de señalar el reducido tamaño muestral, lo que no permite hacer generalizaciones más allá de la muestra que estamos estudiando. En estos términos debe considerarse también la interpretación de la significación estadística obtenida en los análisis. Además, la muestra contó con pocas personas con DI moderada $\sin \mathrm{SD}(\mathrm{N}=2)$, lo que puede haber influido en los resultados, sesgándolos hacia un sobrediagnóstico, debido a la diferencia en los puntos de corte en función del nivel de DI. En un futuro sería interesante ampliar el tamaño muestral, asegurando, además, que las distintas variables de interés (p. e., etiología de la DI, grupo de edad, nivel de funcionamiento, entorno en el que reside la persona, grupos equivalentes de DI moderada y leve) están correctamente representadas.

Por motivos relacionados con el tiempo necesario para realizar una investigación de estas características, el CAMDEX-DS no pudo aplicarse en su totalidad (solo se hizo con aquellos que obtuvieron puntuaciones por debajo de los puntos de corte establecidos por el instrumento).

Por último, y si bien es una limitación sobre la cual no tenemos ningún control, el instrumento incluye alguna pregunta que requiere de actualización, como es el caso de las preguntas ¿Dónde se celebraron los juegos olímpicos del 92? ¿Quién es Lola Flores? Además, es necesario remarcar que alguna pregunta presenta dificultades de comprensión. En el caso del ítem ¿En qué se parecen una fruta y una manzana?, lo que se busca es que la persona mencione la categoría a la que ambos elementos pertenecen (p. e., frutas), pero el verbo 'parecer' lleva a los sujetos a responder indicando aspectos más concretos (p. e., color). Convendría asimismo contar con un mayor número de estudios que exploren la validez discriminante del CAMDEX-DS con muestras de un tamaño adecuado y mayor variabilidad en cuanto a etiología de la DI. Como señalábamos con anterioridad, si bien es importante contar con instrumentos disponibles en lengua española, la urgencia de su necesidad no debiera eclipsar una adecuada adaptación metodológica. El único estudio disponible en la actualidad sobre la validez del CAMDEX-DS (Esteba-Castillo et al., 2013) cuenta con una muestra reducida (N =146) que se reduce aún más si tenemos en cuenta que se ofrecen puntos de corte separados para población con síndrome de Down y población cuya DI no se debe a esta alteración cromosómica; reducción que afecta asimismo a la muestra utilizada para realizar distintos análisis de fiabilidad y validez $(\mathrm{N}=30)$. Igualmente importante sería contar con una muestra en la que distintas variables demográficas que pudieran acelerar el deterioro (p. e., largos procesos de institucionalización en entornos poco o nada estimulantes) estuvieran correctamente representadas. Asimismo, y dada la dificultad de establecer un diagnóstico clínico de deterioro cognitivo en esta población, sería especialmente relevante realizar estudios con esta prueba que tuvieran en cuenta, como señala Flórez (2010), la presencia de posibles fenómenos depresivos en la población con síndrome de Down, que pudieran añadir confusión al diagnóstico. 


\section{DETERIORO COGNITIVO Y TRASTORNO NEURODEGENERATIVO EN PERSONAS CON DISCAPACIDAD INTELECTUAL \\ JUDITH DOMÍNGUEZ GARCÍA Y PATRICIA NAVAS MACHO}

Aun así, este estudio constituye un punto de partida para futuras líneas de investigación. En investigaciones futuras sería necesario realizar la aplicación completa de todo el instrumento en una muestra de mayor tamaño de distintas localizaciones y distintas realidades sociales. En dicha muestra se analizarían de forma más exhaustiva las puntuaciones obtenidas en el CAMDEX-DS completo. Asimismo, otra posible línea de investigación debiera centrarse en el estudio longitudinal de los cambios comportamentales del sujeto, prestando especial atención a cómo estos evolucionan pues pudieran ser indicativos de un estadio inicial de deterioro cognitivo, siendo aún necesario profundizar en la forma en que se desarrolla la enfermedad.

\section{Referencias bibliográficas}

Asociación Americana de Psiquiatría (2013). Manual diagnóstico y estadístico de los trastornos mentales (DSM-5). Arlington: Panamericana.

Ball, S., Dodd, K., Holland, T., Huppert, F. y Treppner, P. (2006). CAMDEX-DS. Cambridge Examination for Mental Disorders of Older people with Down's Syndrome and Others with Intellectual Disabilities. Cambridge: Cambridge University Press.

Ball, S., Holland, T., Huppert, F. A., Treppner, P. y Dodd, K. (2013). Prueba de Exploración Cambridge para la Valoración de los Trastornos Mentales en Adultos con Síndrome de Down o con Discapacidad Intelectual. Madrid, España: TEA Ediciones.

Barrio del Campo, J. A., Árias, M., Ruiz Fernández, M. I. y Vicente Castro, F. (2007). Envejecimiento y discapacidad intelectual: la nueva etapa. INFAD Revista de Psicología, $1(2), 43-56$.

Bell, D. M., Turnbull, A. y KidD, W. B. (2009). Differential diagnosis of dementia in the field of learning disabilities: A case study. British Journal of Learning Disabilities, 37 (1), 56-65. https://doi.org/10.1111/j.1468-3156.2008.00524.x.

Díaz, E., Gómez, L. E. y Alcedo, M. Á. (2016). Síndrome de Down y enfermedad de Alzheimer: factores de riesgo, evaluación e intervención. Revista Española de Discapacidad, 4 (1), 27-51. https://doi.org/10.5569/2340-5104.04.01.02.

Esteba-Castillo, S., Dalmau-Bueno, A., Ribas-Vidal, N., Ribas-Vilà, M., Novell-Alsina, R. y García-Alba, J. (2013). Adaptación y validacion del Cambridge Examination for Mental Disorders of Older People with Down's Syndrome and Others with Intellectual Disabilities (CAMDEX-DS) en población española con discapacidad intelectual. Revista de Neurología, 57 (8), 337-346.

Esteba-Castillo, S., Ribas, N., Baró, M. y Novell, R. (2006). Envejecimiento saludable en personas con síndrome de Down y demencia: necesidad de promover programas de formación y soporte a los usuarios, familias y entidades. Revista Médica Internacional sobre el Sindrome de Down, 10 (2), 25-29.

Eurilngs, H. L., Evenhuis, H. M. y Kengen, M. F. (2006). Dementia Questionnaire for People with Learning Disabilities (DLD). San Antonio, TX: Harcourt Assesment.

Fletcher, R. J., Barnhill, J. y Cooper, S. (2017). Diagnostic Manual-Intellectual Disability ( $2^{\text {nd }}$ edition). Kingston, NY: NAAD Press.

FlóreZ, J. (2010). Enfermedad de Alzheimer y síndrome de Down. Revista Sindrome de Down, 27, 63-76.

Heller, T. (2009). Envejecimiento y discapacidad intelectual. Siglo Cero, 40 (1), 67-78. 


\section{DETERIORO COGNITIVO Y TRASTORNO NEURODEGENERATIVO EN PERSONAS CON DISCAPACIDAD INTELECTUAL \\ JUDITH DOMÍNGUEZ GARCÍA Y PATRICIA NAVAS MACHO}

Huppert, F. A., Mountjoy, C. Q., Roth, M. y Tym, E. (1998). Camdex-R: The Cambridge Examination for Mental Disorders of the Elderly. Cambridge: Cambridge University Press.

Instituto Nacional de Estadística (2008). Encuesta sobre Discapacidad, Autonomía personal y situaciones de Dependencia, EDAD. Portal Mayores, 87, 12.

Janicki, M. P., Heller, T., Seltzer, G. B. y Hogg, J. (1996). Practice guidelines for the clinical assessment and care management of Alzheimer's disease and other dementias among adults with intellectual disability. Journal of Intellectual Disability Research, 40 (4), 374-382.

Janicki, M. P. y Dalton, A. J. (2000). Prevalence of dementia and impact on intellectual disability services. Mental Retardation, 38, 3, 276-288.

Livingston, G., King, M., Hassiotis, A. y STYdom, A. (2007). Prevalence of dementia in intellectual disability using different diagnostic criteria. British Journal of Psychiatry, 191, 150-157.

Makary, A. T., Testa, R., Tonge, B. J., Einfeld, S. L., Mohr, C. y Gray, K. M. (2015). Association between adaptive behaviour and age in adults with down syndrome without dementia: Examining the range and severity of adaptive behaviour problems. Journal of Intellectual Disability Research, 59 (8), 689-702.

McCarron, M., McCallion, P., Reilly, E. y Mulryan, N. (2014). A prospective 14 year longitudinal followup of dementia in persons with Down syndrome. Journal of Intellectual Disability Research, 58 (1), 61-70.

Moran, J. A., Rafi, M. S., Keller, S. M., Singh, B. K. y Janicki, M. P. (2013). The National Task Group on intellectual disabilities and dementia practices consensus recommendations for the evaluation and management of dementia in adults with intellectual disabilities. Mayo Clinic Proceedings, 88 (8), 831-840. https://doi.org/10.1016/j.mayocp.2013.04.024.

Oliver, C., Adams, D. y Kalsy, S. (2008). Ageing, dementia and people with intellectual disability. En B. Woods y L. Clare (Eds.), Handbook of the Clinical Psychology of Ageing (pp. 341-349). Chichester, Reino Unido: John Wiley \& Sons, Ltd.

Presson, A. P., Partyka, G., Jensen, K. M., Devine, O. J., Rasmussen, S. A., McCabe, L. L. y McCABE, E. R. B. (2013). Current estimate of Down syndrome population prevalence in the United States. The Journal of Pediatrics, 163 (4), 1163-1168.

Sheehan, R., Sinai, A., Bass, N., Blatchford, P., Bohnen, I., Bonell, S. y Mukherji, K. (2015). Dementia diagnostic criteria in Down syndrome. International Journal of Geriatric Psychiatry, 30 (8), 857-863.

Silverman, W. P., Zigman, W. B., Krinsky-McHale, S. J., Ryan, R. y Schupf, N. (2013). Intellectual disability, mild cognitive impairment, and risk for dementia. Journal of Policy and Practice in Intellectual Disabilities, 10 (3), 245-251.

Tassé, M. J., Navas, P., Havercamp, S. M., Benson, B. A., Allain, D. C., Manickam, K. y DaVIS, S. (2016). Psychiatric conditions prevalent among adults with Down syndrome. Journal of Policy and Practice in Intellectual Disabilities, 13 (2), 173-180.

Walsh, D. M., Doran, E., Silverman, W., Tournay, A., Movsesyan, N. y LotT, I. T. (2015). Rapid assessment of cognitive function in Down syndrome across intellectual level and dementia status. Journal of Intellectual Disability Research, 59 (11), 1071-1079. 